\title{
- Letters to the editor
}

\section{Use of epidemiological principles in research and scientific publications - future perspective}

Wagle RR, MD, PhD

Institute of Medicine, Tribhuwan University, Kathmandu, Nepal

Dear Editor,

Epidemiology has pervaded clinical and medical sciences these days. On one hand, the clinicians are measuring everything exactly and reporting comfortably in numbers. On the other, these very scientists are not encouraged to talk about their tacit knowledge of clinical experience and acumen. Mystification of clinical work and judgment has been labeled as an avoidable nuisance. But at the same time, epidemiology has been thought as distinct from clinical science and research. As a consequence, discussion on epidemiological methods and issues of causation of disease has been confined within the epidemiology departments and schools of public health.

Working together for mutual benefit is imperative in today's context for the advancement of knowledge and its incorporation in clinical practice. Observation, description and analysis are the components of epidemiology. To produce a generalisable knowledge from time and place specific observations is the basic tenet of research. The future perspective of clinical research depends on the mutual understanding and combined efforts of the clinicians and the epidemiologists.

Source of support: nil. Conflict of interest: none 